\title{
OPTIMIZACIÓN DEL TRABAJO EN UN CICLO BRAYTON CON IRREVERSIBILIDADES
}

\author{
Manuel Malaver
}

\begin{abstract}
Resumen
Considerando las irreversibilidades asociadas a los ciclos de turbina de gas reales, en esta investigación se optimiza el trabajo neto en un ciclo Brayton para un motor de turbina a gas en términos de la relación de trabajo de retroceso. Los resultados obtenidos permiten deducir una expresión para la relación de trabajo de retroceso que depende de los límites de temperatura máxima y mínima del ciclo, de las eficiencias adiabáticas del compresor y la turbina del ciclo Brayton y es independiente de la sustancia de trabajo utilizada.
\end{abstract}

Palabras clave: Ciclo Brayton; compresor; eficiencia adiabática; irreversibilidades; relación de trabajo de retroceso; turbina.

\begin{abstract}
Considering the irreversibilities associated with the real cycles of gas turbine, in this paper the work is optimized in a cycle Brayton for an engine of turbine to gas in terms of the relation of work of setback. The obtained results allow to deduce an expression for the relation of work of setback that depends on the limits of maximum temperature and minim of the cycle, on the adiabatic efficiencies of the compressor and the turbine of the cycle Brayton and is independent from the substance of work used.
\end{abstract}

Keywords: Brayton cycle; compressor; adiabatic efficiency; irreversibilities; relation of work of setback; turbine.

Recibido: 12 de Diciembre del 2011 • Aprobado: 21 de Mayo del 2012

\section{INTRODUCCIÓN}

En termodinámica existen dos áreas fundamentales de aplicación, la generación de potencia y la refrigeración. Su estudio se hace a través de ciclos termodinámicos, los cuales se dividen en ciclos de potencia y de refrigeración entre los que se incluyen el ciclo de Carnot, Otto, Diesel, Dual, Rankine, Brayton, Stirling y Ericsson (Cengel y Boles, 2006; Van Wylen et al., 2004; Wark y Richards, 2001 ).

Los ciclos termodinámicos se pueden clasificar en ciclos cerrados y abiertos. En los ciclos cerrados la sustancia de trabajo se regresa a su estado inicial al final del ciclo y debe recircularse. Los motores de combustión interna llamados también máquinas térmicas, funcionan de tal manera que los gases de combustión se escapan y se reemplazan por una nueva mezcla aire-combustible al terminar cada ciclo.

La operación estándar de los motores de combustión interna se realiza en el modo de presión limitada, o en el modo de volumen limitado (Russel y Adebiyi, 1997). Las turbinas de gas son un ejemplo del primer modo mientras que los motores de combustión interna como el motor Otto y Diesel se diseñan para funcionar en el modo de volumen limitado (Cengel y Boles, 2006; Russel y Adebiyi, 1997).

Los ciclos de potencia han sido objeto de valiosas idealizaciones que han sido útiles para el diseño ( Herrera et al., 2008; Malaver, 2008; Cengel y Boles, 2006; Urrecheaga y Malaver, 2003; Chen et al., 2002; Wark y Richards, 2001; Guzmán-Vargas y Angulo-Brown, 1998; Jones y Dugan, 1997; Leff, 1987; Curzon y Ahlborn, 
1975), aún cuando las plantas se desvían de estas simplificaciones, porque máquinas y procesos reales son imperfectos y no se rigen fielmente por los esquemas ideales. Es por esta razón que modelar un sistema que trate de reproducir la realidad constituye un reto para la ingeniería, siempre expuesta a discusiones por las suposiciones y aproximaciones que se incorporan (Herrera et al., 2008 y Burghardt, 1984).

El análisis de los ciclos reales de los motores de combustión interna es complicado debido a la presencia de fenómenos e irreversibilidades como la fricción y la rapidez de los procesos de expansión-compresión que impiden establecer condiciones de equilibrio termodinámico, por lo que sí se quiere hacer manejable el estudio de un ciclo se deben hacer algunas simplificaciones (Cengel y Boles, 2006). Cuando a un ciclo real se le eliminan todos los problemas internos complejos se obtiene un ciclo constituido por una secuencia de pasos reversibles. Un ciclo que cumple con estas condiciones recibe el nombre de ciclo ideal. Para una turbina de gas, el ciclo ideal es el ciclo Brayton.

Los ciclos de turbina de gas reales difieren del ciclo Brayton ideal debido a que ocurre una disminución de presión durante los procesos de adición y eliminación térmica y por irreversibilidades como la fricción y las condiciones de operación de estos dispositivos (Cengel y Boles, 2006).
En esta investigación, se analizan los efectos de las irreversibilidades asociadas a la relación de trabajo de retroceso de un ciclo Brayton ideal cuando se tienen en cuenta las eficiencias adiabáticas del compresor y la turbina. En la Sección 2 se hace una descripción de un ciclo Brayton ideal para una turbina de gas y se mencionan algunas de sus aplicaciones más importantes. En la Sección 3 se hace referencia a la desviación del comportamiento real del compresor y la turbina respecto del comportamiento isentrópico idealizado del ciclo Brayton. En la Sección 4 se presenta la metodología planteada para el presente trabajo de investigación. En la Sección 5 se muestra como varía el trabajo neto con la relación de trabajo de retroceso y la dependencia de la relación de trabajo de retroceso máxima con respecto a las variables involucradas en el problema. La sección 6 contiene las conclusiones de este trabajo.

\section{EL CICLO DE BRAYTON PARA UNA TURBINA DE GAS}

El ciclo Brayton fue propuesto por primera vez por George Brayton para emplearlo en un motor que quemaba aceite que él mismo diseñó en 1870 (Cengel y Boles, 2006; Jones y Dugan,1997). Las turbinas de gas usualmente operan en un ciclo abierto, como se ilustra en la Figura 1.

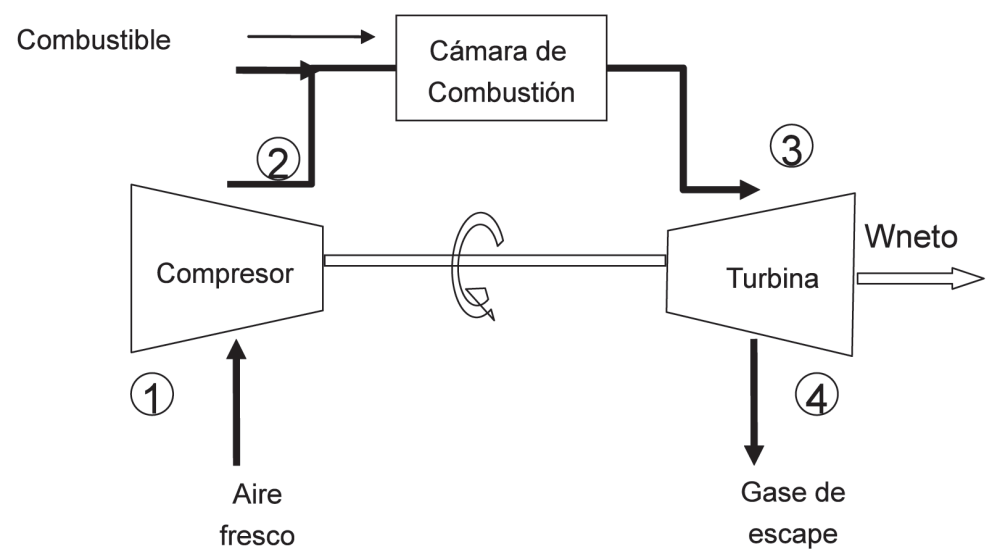

Figura 1. Esquema de un motor de turbina de gas de ciclo abierto. 
El aire en condiciones ambientales se introduce dentro de un compresor axial o centrífugo donde su temperatura y presión se eleva. El aire de alta presión sigue hacia la cámara de combustión donde el combustible se quema a presión constante. Luego los gases de alta temperatura que resultan entran a la turbina, donde se expanden hasta la presión atmosférica, de manera tal que producen potencia. Los gases de escape que salen de la turbina se expulsan hacia fuera ( Dugan y Jones, 1997).

Debido a la complejidad de este ciclo de potencia, los ingenieros se ven en la necesidad de trabajar con modelos idealizados, por lo que se han de establecer las siguientes aproximaciones, llamadas suposiciones de aire estándar (Cengel y Boles, 2006):

- La sustancia de trabajo es un fluido que circula de modo continuo en un circuito cerrado y se comporta como un gas ideal.

- Todos los procesos que integran el ciclo son reversibles.

- El proceso de combustión se reemplaza por un proceso de adición térmica desde una fuente externa.

- El proceso de escape se sustituye por un proceso de eliminación térmica que regresa la sustancia de trabajo a su estado inicial.

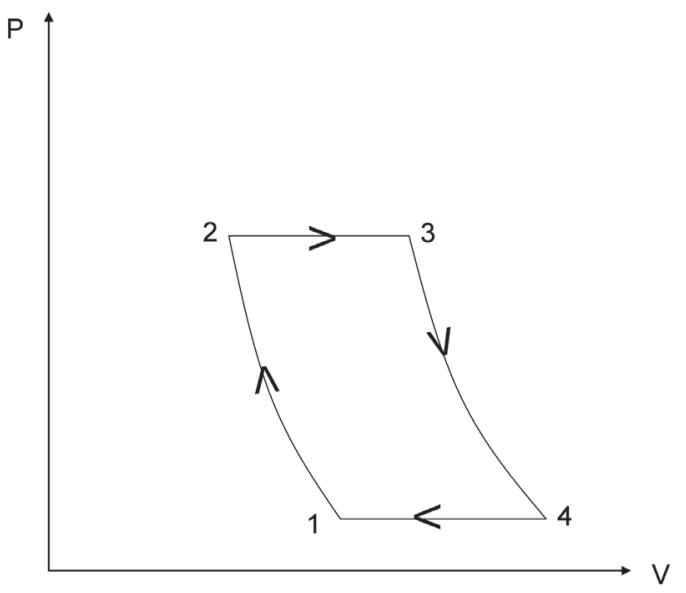

Figura 2. Diagrama P-V de un ciclo Brayton.
Con estas suposiciones el ciclo Brayton (Wark y Richards, 2001) se compone de los siguientes procesos reversibles (ver Figura 2 y 3 ):

$$
\begin{aligned}
& 1 \rightarrow 2 \text { ) Compresión isentrópica } \\
& 2 \rightarrow 3 \text { ) Calentamiento isobárico } \\
& 3 \rightarrow 4 \text { ) Expansión isentrópica } \\
& 4 \rightarrow 1) \text { Enfriamiento isobárico }
\end{aligned}
$$

De acuerdo con las aproximaciones realizadas para un ciclo Brayton y utilizando la convención que define el trabajo durante un proceso internamente reversible asociado a sistemas cerrados como $W=-\int P d v$ (Wark y Richards, 2001), el trabajo neto del ciclo estará dado por:

$$
W_{\text {neto }}=W_{1 \rightarrow 2}+W_{2 \rightarrow 3}+W_{3 \rightarrow 4}+W_{4 \rightarrow 1}
$$

donde $W_{l \rightarrow 2}$ es $W_{l \rightarrow 2}=C_{v}\left(T_{2}-T_{1}\right)$

$$
W_{2 \rightarrow 3}=R\left(T_{2}-T_{3}\right)
$$

$W_{3 \rightarrow 4}=C_{v}\left(T_{4}-T_{3}\right)$

$W_{4 \rightarrow 1}=R\left(T_{4}-T_{1}\right)$

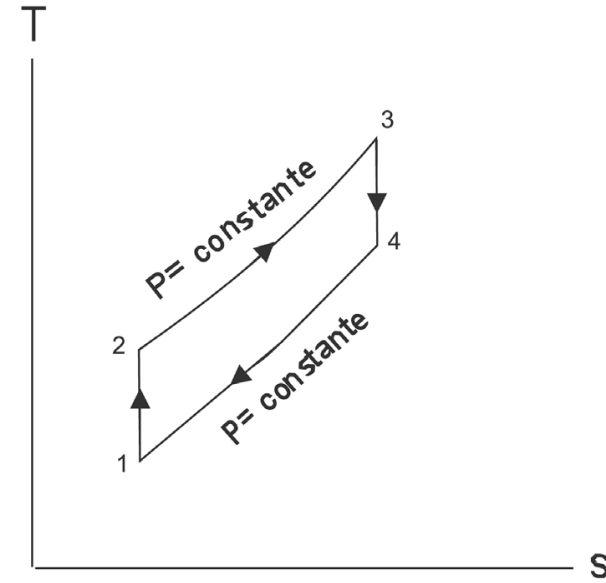

Figura 3. Diagrama T-S de un ciclo Brayton. 
por lo que:

$W_{\text {neto }}=C_{v}\left(T_{2}-T_{1}\right)+R\left(T_{2}-T_{3}\right)+C_{V}\left(T_{4}-T_{3}\right)+$ $R\left(T_{4}-T_{1}\right)$

Esta expresión se puede expresar de la siguiente manera:

$W_{\text {neto }}=C_{v}\left[\left(T_{4}-T_{1}\right)+\left(T_{2}-T_{3}\right)\right]+R\left[\left(T_{4}-T_{1}\right)+\left(T_{2}-T_{3}\right)\right]$

Agrupando términos semejantes y extrayendo $T_{1}$ como factor común queda que:

$W_{\text {neto }}=C_{p} T_{1}\left[\left(T_{4} / T_{1}-1\right)+\left(T_{2} / T_{1}-T_{3} / T_{1}\right)\right]$

En el ciclo Brayton se ha definido la relación de presiones como $r_{p}=P_{2} / P_{1}$ y teniendo en cuenta que para el proceso 1-2 se cumple que:

$\frac{T_{1}^{C_{p} / R}}{P_{1}}=\frac{T_{2}^{C_{p} / R}}{P_{2}}$

se obtiene que: $\frac{T_{2}}{T_{1}}=r_{p}^{\frac{\gamma-1}{\gamma}}$

donde: $\quad \gamma=\frac{C_{P}}{C_{V}}$

Del mismo modo y tomando en cuenta $P_{1}=P_{4}$ y $P_{2}=P_{3}$ también se demuestra que:

$\frac{T_{3}}{T_{4}}=r_{p}^{\frac{\gamma-1}{\gamma}}$

Reemplazando (10) y (12) en la expresión (8) queda que:

$$
W_{\text {neto }}=C_{p} T_{1}\left[\left(\frac{T_{3}}{T_{1}} r_{p}^{\frac{1-\gamma}{\gamma}}-1\right)+\left(r_{p}^{\frac{\gamma-1}{\gamma}}-\frac{T_{3}}{T_{1}}\right)\right]
$$

El trabajo $\mathrm{W}_{1 \rightarrow 2}$ y $\mathrm{W}_{3 \rightarrow 4}$ se ha definido como el trabajo de entrada al compresor y el trabajo de salida de la turbina, respectivamente y la relación entre el trabajo del compresor y el trabajo de la turbina $r_{t r}=-\mathrm{W}_{l \rightarrow 2} / \mathrm{W}_{3 \rightarrow 4}=-\mathrm{W}_{C}$ / $\mathrm{W}_{T}$ recibe el nombre de relación del trabajo de retroceso o relación de acoplamiento turbinacompresor (Cengel y Boles, 2006; Wark y Richards, 2001).

Las turbinas de gas han tenido una gran aplicación en la propulsión de buques de guerra y del sector marítimo comercial (Woodyard, 2004), así como también en la propulsión de aviones y la generación de energía eléctrica (Cengel y Boles, 2006). Los principales candidatos para la propulsión a turbina a gas en el transporte marítimo son los buques de crucero, ferrys de pasajeros, los buques que transportan gas natural licuado y los portacontenedores (Woodyard, 2004). Cuando se utilizan en propulsión de aviones, la turbina de gas produce suficiente potencia para accionar el compresor y los gases de escape de alta velocidad son los responsables de producir el empuje necesario para impulsar la aeronave (Cengel y Boles, 2006; Burghardt, 1984). Las centrales eléctricas de turbina de gas son empleadas por la industria de generación de energía eléctrica en emergencias, debido a su bajo costo y rápido tiempo de respuesta. El ciclo de turbina de gas también puede ejecutarse como un ciclo cerrado para ser utilizado en centrales nucleoeléctricas, en la que se emplean como sustancias de trabajo gases como el helio o el argón (Cengel y Boles, año 2006).

Es importante resaltar el hecho de que en las plantas eléctricas de turbina a gas la relación de trabajo de retroceso suele ser muy alta (Cengel y Boles, 2006; Wark y Richards, 2001), debido a que más de la mitad de la salida de trabajo de la turbina se utiliza para activar el compresor, por lo que una central eléctrica requiere de grandes turbinas para cumplir con los requerimientos de potencia del compresor.

Entre las principales ventajas que tiene el utilizar las plantas de propulsión a turbina a gas se encuentran las siguientes: a) alta potencia y altas relaciones de compresión; b) bajo ruido y vibración; c) fácil instalación y bajos costos de mantenimiento; c) bajas emisiones contaminantes, menores que en los motores Diesel convencionales (Woodyard, 2004). 


\section{IRREVERSIBILIDADES DEL COMPRESOR Y LA TURBINA}

El trabajo isoentrópico asociado a los compresores y turbinas que funcionan de manera adiabática es un modelo con el que se pueden comparar los equipos reales (Wark y Richards, 2001). La desviación del comportamiento real de estos dispositivos respecto del comportamiento isentrópico idealizado se puede explicar si se utilizan las eficiencias adiabáticas de la turbina y el compresor (Cengel y Boles, 2006; Wark y Richards, 2001) definidas como:

$$
\begin{array}{r}
\eta_{T}=\frac{W_{\text {sal }}}{W_{s, s a l}} \\
\text { y } \quad \eta_{C}=\frac{W_{s, e n t}}{W_{e n t}}
\end{array}
$$

Donde $W_{\text {sal }}$ es el trabajo de salida real, $W_{s, s a l}$ es el trabajo de salida isentrópico, $W_{s, \text { ent }}$ es el trabajo de entrada isentrópico y $W_{e n t}$ es el trabajo real necesario a la entrada donde el trabajo de salida isentrópico y el trabajo de entrada isentrópico vendrán dados por (4) y (2), respectivamente.

La expresión para el trabajo neto se puede reescribir en función de las irreversibilidades en el compresor y la turbina, por lo que la ecuación (1) queda como:

$$
W_{\text {neto }}=W_{s, \text { ent }} / \eta_{C}+\eta_{T} W_{s, \text { sal }}+W_{2 \rightarrow 3}+W_{4 \rightarrow 1}
$$

Teniendo en cuenta que $\mathrm{W}_{1 \rightarrow 2}=\mathrm{W}_{s, \text { ent }}, \mathrm{W}_{3 \rightarrow 4}$ $=\mathrm{W}_{s, \text { sal }}$ y sustituyendo las ecuaciones (3), (5) en (16), la expresión $W_{\text {neto }}$ (Manrique y Cárdenas, 1981) se escribe como:

$$
W_{\text {neto }}=-\eta^{*} C_{p} T_{1}\left[\frac{T_{3}}{T_{1}} \eta_{T}-\frac{r_{p}^{\frac{\gamma-1}{\gamma}}}{\eta_{C}}\right]
$$

en la que:

$$
\eta^{*}=1-\frac{1}{r_{p}^{\frac{\gamma-1}{\gamma}}}
$$

es la expresión para la eficiencia térmica de un ciclo Brayton ideal (Cengel y Boles, 2006; Van Wylen et al.,2004; Wark y Richards, 2001).

Teniendo en cuenta (17) y el hecho que el valor de:

$$
Q_{a b s}=Q_{2 \rightarrow 3}=C_{P}\left(T_{3}-T_{2}\right) \text {, }
$$

se obtiene una expresión para la eficiencia en la que se incluye $\eta_{T}$ y $\eta_{C}$ dada por:

$$
\eta=\eta^{*} \frac{\frac{T_{3}}{T_{1}} \eta_{T}-\frac{r_{p}^{\frac{\gamma-1}{\gamma}}}{\eta_{C}}}{\frac{T_{3}}{T_{1}}-1-\frac{r_{p}^{\frac{\gamma-1}{\gamma}}-1}{\eta_{C}}}
$$

La eficiencia térmica depende también de las eficiencias adiabáticas del compresor y la turbina y de las temperaturas a la entrada de la turbina y a la entrada del compresor (Manrique y Cardenas, 1981).

A partir de (17), se tiene que en el punto de trabajo o potencia máxima:

$$
r_{o p t}=\left(\eta_{c} \eta_{t} \frac{T_{3}}{T_{1}}\right)^{\frac{\gamma}{2(\gamma-1)}}
$$

Del mismo modo, de acuerdo con la ecuación (15), para máxima eficiencia se tiene:

$\frac{d \eta}{d r_{\text {opt }}}=0$, de donde se deduce:

$$
r_{\text {opt }}=\left\{\frac{\frac{T_{3}}{T_{1}} \eta_{C}+1-\eta_{C}}{1+\left[\frac{\left(T_{3} / T_{1}-1\right)\left(\eta_{C} T_{3} / T_{1}+1-\eta_{C}-\eta_{C} \eta_{T} T_{3} / T_{1}\right)}{\eta_{T} T_{3} / T_{1}}\right]^{1 / 2}}\right\}^{\gamma / \gamma-1}
$$

En la Figura 4 se representa la variación del trabajo neto y de la eficiencia térmica en función de la relación de presiones. Las líneas punteadas señalan la relación óptima de presiones para que la turbina de gas tenga máxima eficiencia 

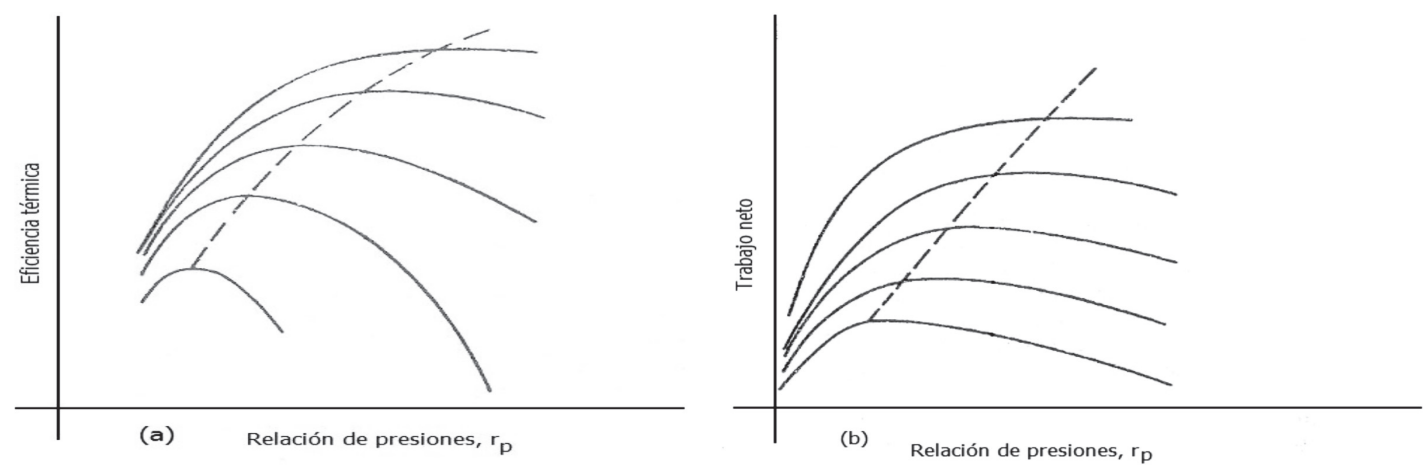

Figura 4. Eficiencia térmica y trabajo neto en función de la relación de presiones. Cada curva representa un valor dado de temperatura $T_{3}$.

Fuente: El autor.

o el trabajo neto alcance su máximo valor. De acuerdo con las ecuaciones (20) y (21), es de esperarse que una misma relación de presiones no produzca simultáneamente la máxima eficiencia y el máximo trabajo, para una máxima eficiencia se requiere un valor específico de la relación de presiones y para una máxima potencia o trabajo se requiere otra, es por esto que las turbinas de gas se diseñan generalmente para máxima potencia, máxima economía o un equilibrio entre estos dos parámetros (Manrique y Cardenas, 1981). En efecto, un cálculo simple con $T_{1}=300 \mathrm{~K} ; T_{3}$ $=1200 \mathrm{~K} ; \eta_{T}=0.85 ; \eta_{C}=0.80 ; \gamma=1.4$ para aire como sustancia de trabajo, permite obtener para el trabajo máximo $r_{o p t}=6 \mathrm{y}$ para máxima eficiencia $r_{\text {opt }}=10$.

\section{METODOLOGÍA}

Utilizando las suposiciones de aire estándar que permiten estudiar ciclos de potencia de gases, se analizó la influencia de la relación de presiones, temperatura máxima y temperatura mínima del ciclo en el trabajo neto y en la relación de trabajo de retroceso en un ciclo Brayton. La temperatura de entrada al compresor o temperatura mínima era de $300 \mathrm{~K}$ y la temperatura máxima o temperatura de entrada a la turbina de 1500 , 2000 y $2500 \mathrm{~K}$, respectivamente. La presión al inicio del proceso de compresión era de 100 $\mathrm{kPa}$. El estudio se realizo para tres sustancias de trabajo diferentes, que en este caso fueron helio, hidrógeno y aire. Se espera que los resultados de este trabajo puedan utilizarse para determinar la factibilidad del hidrógeno como combustible en el sector marítimo internacional al comparar su eficacia con otros fluidos disponibles en el mercado actual. Se consideró que las capacidades caloríficas varían con la temperatura y que las eficiencias adiabáticas del compresor y la turbina son del $75 \%$ y $80 \%$, respectivamente.

\section{ANÁLISIS}

En el Cuadro 1 se representan los valores de la relación de presiones, trabajo neto y relación de trabajo de retroceso para Helio, Hidrógeno y aire. En todos los casos se ha considerado que $\mathrm{T} 1=300 \mathrm{~K}$ y T3 = $2000 \mathrm{~K}$. Para cada una de las sustancias de trabajo utilizadas se observa que el trabajo neto depende de la sustancia de trabajo que se esté utilizando y aumenta a medida que se incrementa la relación de trabajo de retroceso, alcanza un máximo y luego empieza a disminuir, comportamiento que se representa en las Figuras 5, 6 y 7. De acuerdo al Cuadro 1, para el caso específico del helio el trabajo máximo se alcanza cuando el valor del cociente $W_{C} / W_{T}$ es 51.19. Para el hidrógeno y aire se obtienen resultados similares, pues el trabajo se optimiza cuando la relación de acoplamiento adquiere los valores de 50.11 y 50.76, respectivamente, lo cual indica que el trabajo máximo no va a depender de la 

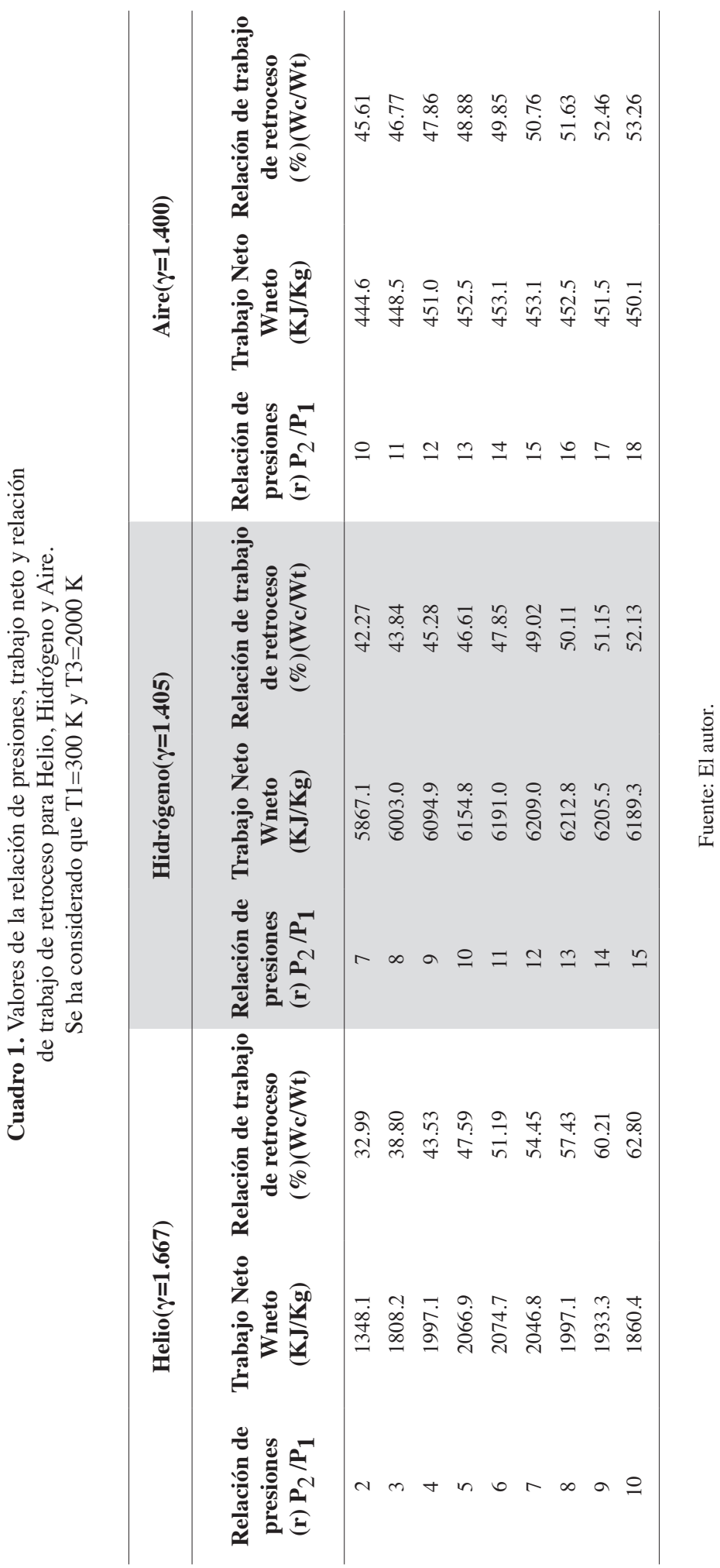


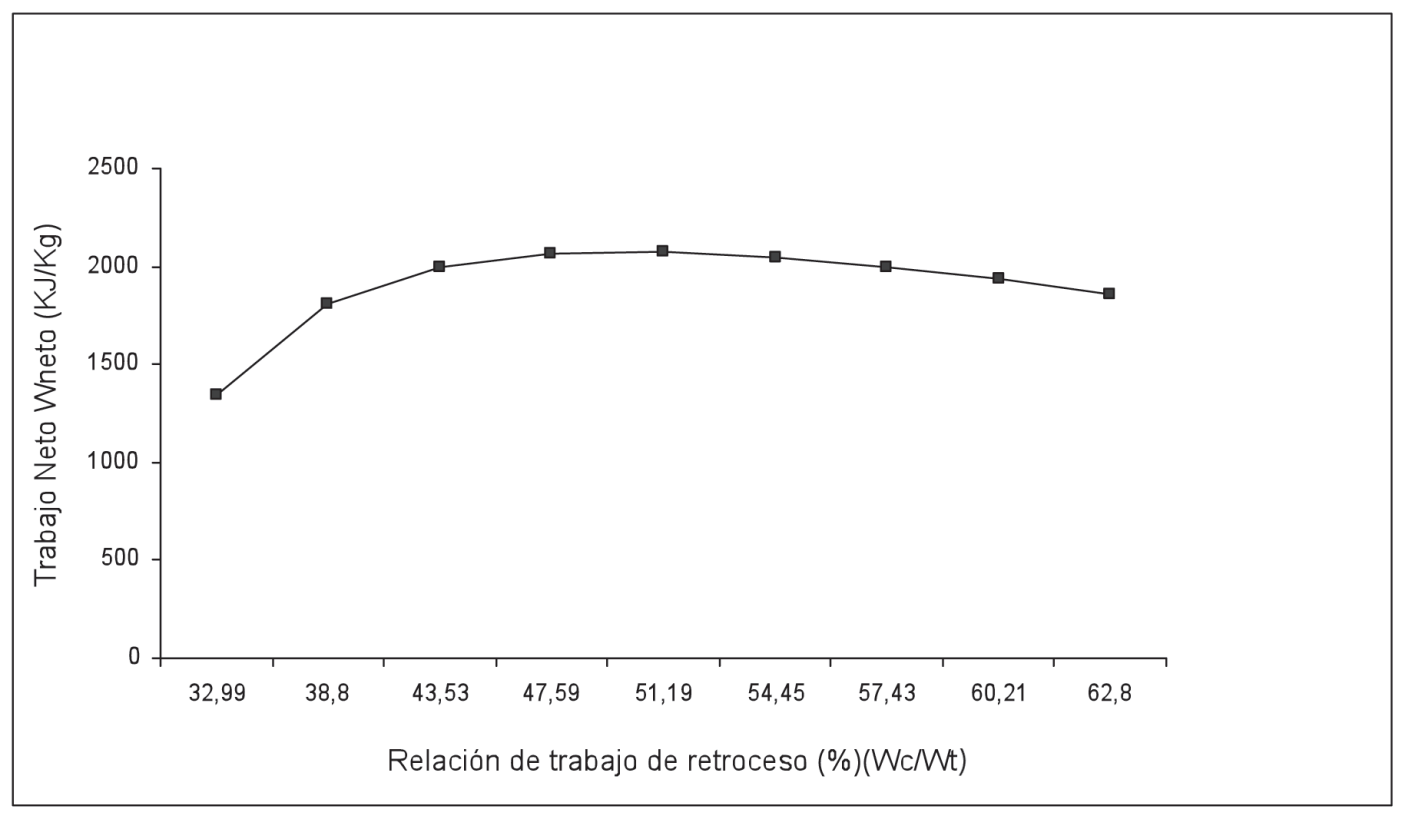

Figura 5. Variación del trabajo neto en $\mathrm{KJ} / \mathrm{Kg}$ versus la relación de trabajo de retroceso para el helio.

Fuente: El autor.

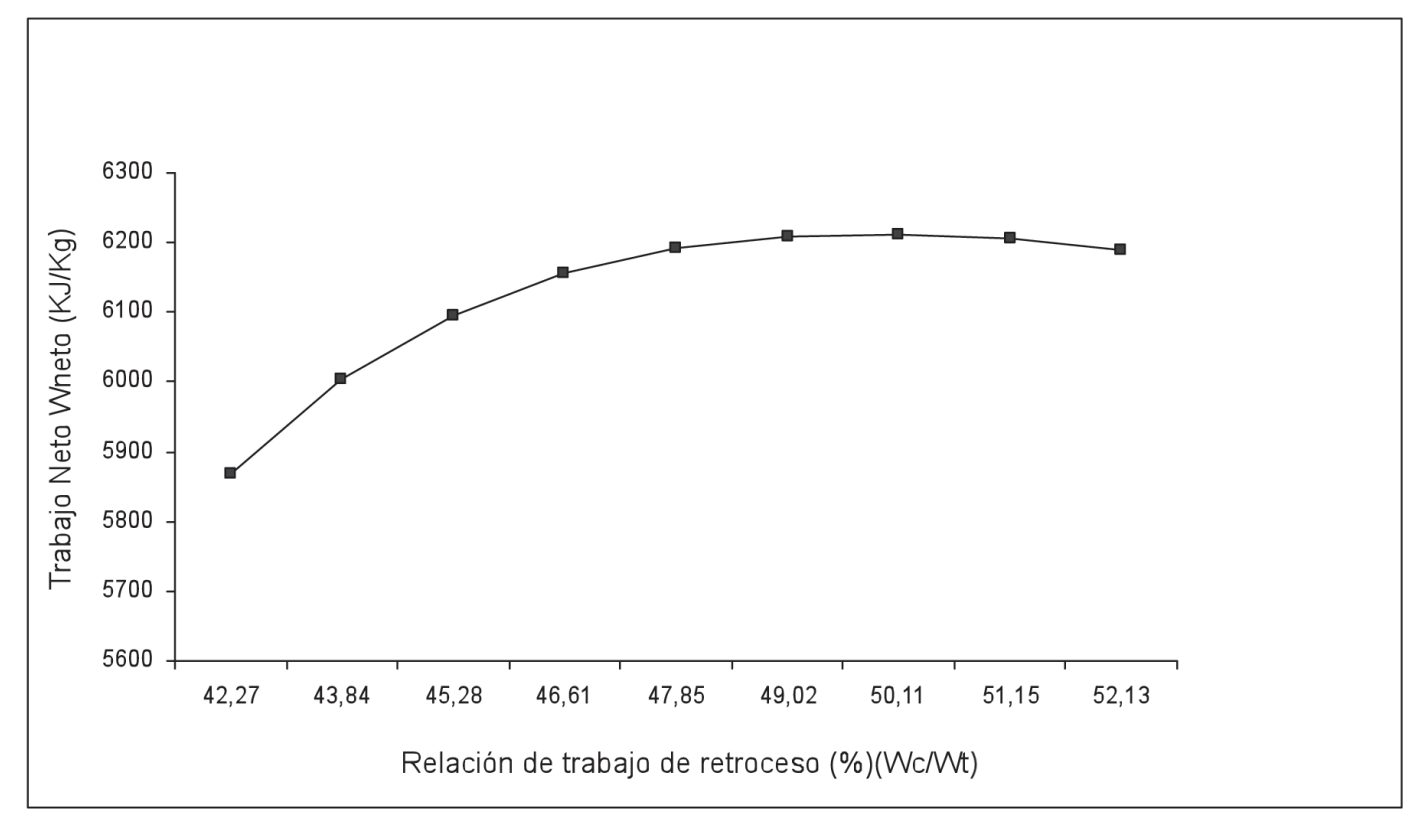

Figura 6. Variación del trabajo neto en $\mathrm{KJ} / \mathrm{Kg}$ versus la relación de trabajo de retroceso para el hidrógeno.

Fuente: El autor. 


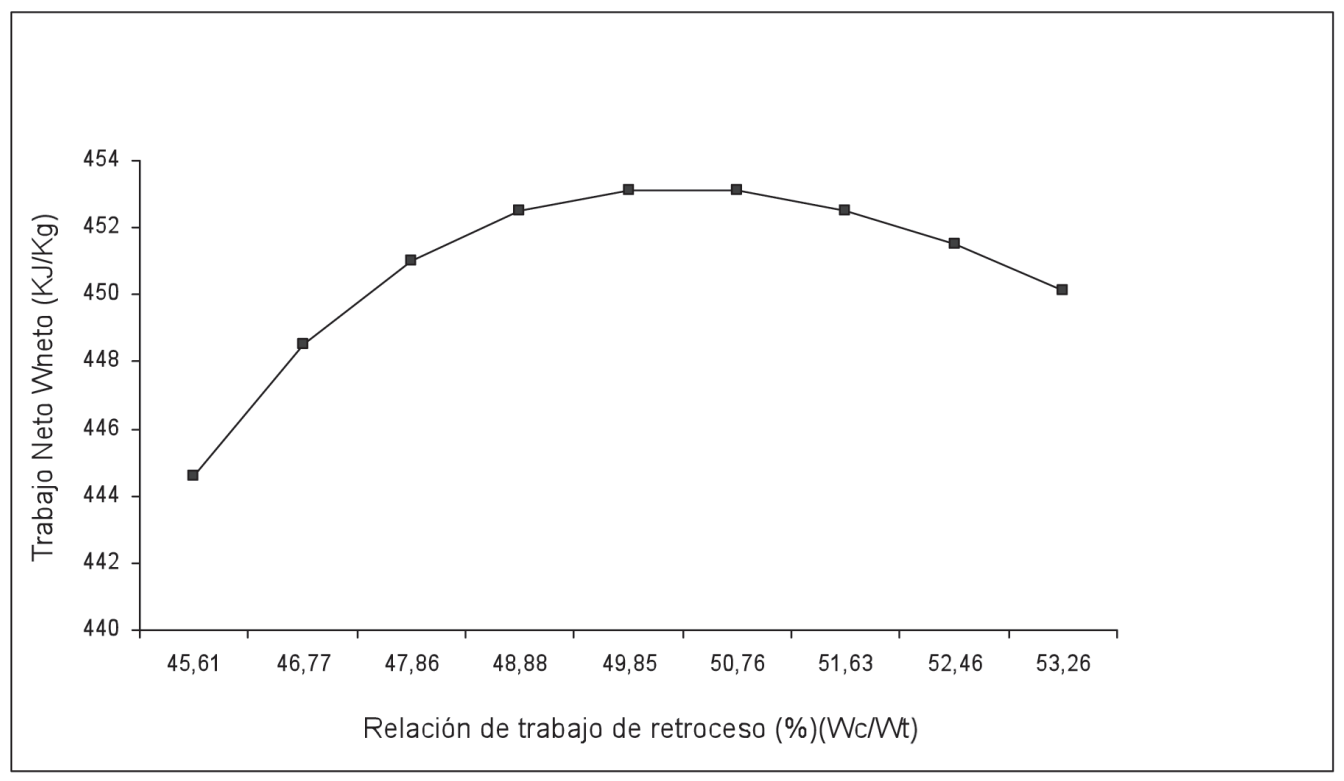

Figura 7. Variación del trabajo neto en $\mathrm{KJ} / \mathrm{Kg}$ versus la relación de trabajo de retroceso para el aire.

Fuente: El autor.

sustancia de trabajo pues es aproximadamente el mismo para helio, hidrógeno y aire. Este comportamiento no se observa con la relación de presiones, la cual, cuando se maximiza el trabajo, va a depender del tipo de fluido que se esté usando (Cengel y Boles, 2006; Wark y Richards, 2001; Jones y Dugan, 1997 y Russel y Adebiyi, 1997). En efecto (ver Cuadro 1), para el helio el máximo se alcanza cuando la relación de presiones $r_{p}=P_{2} / P_{1}$ es 7 y para el hidrógeno cuando $r_{p}=13$. Basándose en estos resultados, se puede deducir una expresión para la relación de trabajo de retroceso en función de la sustancia de trabajo y de la relación de presiones.

De acuerdo a la definición de:

$r_{t r}=-W_{\text {ent }} / W_{\text {sal }}$ se tiene que:

$$
\frac{W_{e n t}}{W_{\text {sal }}}=\frac{C_{v}\left(T_{2}-T_{1} / \eta_{C}\right.}{C_{v}\left(T_{3}-T_{4}\right) \eta_{T}}=\frac{1}{\eta_{C} \eta_{T}} \frac{T_{1}\left(\frac{T_{2}}{T_{1}}-1\right)}{T_{3}\left(1-\frac{T_{4}}{T_{3}}\right)}
$$

Sustituyendo (10) y (12) en (22) se obtiene:

$$
r_{t r}=\frac{W_{1 \rightarrow 2}}{W_{3 \rightarrow 4}}=\frac{1}{\eta_{C} \eta_{T}} \frac{T_{1}}{T_{3}} \frac{\left(r_{p}^{\frac{\gamma-1}{\gamma}}-1\right)}{\left(r_{p}^{\frac{1-\gamma}{\gamma}}-1\right)}
$$

por lo que (23) se puede expresar como:

$$
r_{t r}=\frac{1}{\eta_{C} \eta_{T}} r_{p} \frac{\gamma-1}{\gamma} \frac{T_{1}}{T_{3}}
$$

De acuerdo con (24) la relación de trabajo de retroceso para un ciclo de Brayton real es función de la relación de presiones, de la sustancia de trabajo, la temperatura de entrada al compresor $T_{1}$ y temperatura de entrada a la turbina $T_{3} \mathrm{y}$ de las eficiencias adiabáticas del compresor y la turbina.

La expresión (24) se puede sustituir en (17) con el fin de determinar una relación de trabajo de retroceso para que un motor de turbina a gas suministre un trabajo máximo. La expresión (17) también se escribe en función de la relación de trabajo de retroceso y queda que: 


$$
W_{\text {neio }}=-\left(1-\frac{1}{r_{t r} \eta_{C} \eta_{T}} \frac{T_{1}}{T_{3}}\right) C_{p} T_{1}\left[\frac{T_{3}}{T_{1}} \eta_{T}-\frac{1}{\eta_{C}} \frac{T_{3}}{T_{1}} r_{t r} \eta_{C} \eta_{T}\right]
$$

El trabajo neto en un ciclo Brayton real es función de la relación de trabajo de retroceso, de las temperaturas máxima y mínimas del ciclo y de las eficiencias adiabáticas del compresor y la turbina, a diferencia de la expresión propuesta por Guzmán-Vargas y Angulo-Brown (1998) en la que el trabajo es función de las temperaturas del ciclo y de los procesos $2 \rightarrow 3$ y $4 \rightarrow 1$ (ver Figura 2) y no se muestra la dependencia con la relación de trabajo de retroceso ni con las eficiencias adiabáticas.

En el punto de trabajo máximo se tiene que:

$$
\frac{d W_{\text {neto }}}{d r_{t r}}=-C_{P} T_{1} \frac{d}{d r_{t r}}\left(1-\frac{1}{r_{t r} \eta_{C} \eta_{T}} \frac{T_{1}}{T_{3}}\right)_{1}\left[\frac{T_{3}}{T_{1}} \eta_{T}-\frac{1}{\eta_{C}} \frac{T_{3}}{T_{1}} r_{t r} \eta_{C} \eta_{T}\right]=0
$$

por lo que:

$$
r_{\text {trmax }}=\frac{\left(\frac{1}{\eta_{C}}-1\right) \frac{T_{1}}{T_{3}} \frac{1}{\eta_{T}}+\left[\left(1-\frac{1}{\eta_{C}}\right)^{2}\left(\frac{T_{1}}{T_{3}}\right)^{2} \frac{1}{\eta_{T}{ }^{2}}+\frac{4 T_{1}}{T_{3} \eta_{C} \eta_{T}}\right]^{1 / 2}}{2}
$$

Evaluando (27) en el límite cuando: $\eta_{T} \rightarrow 1$ y $\eta_{C} \rightarrow 1$ se obtiene la ecuación para:

$$
r_{\text {trmax }}=\sqrt{\frac{T_{1}}{T_{3}}}
$$

donde se consideran que los procesos de expansión y compresión son isentrópicos lo que significa que las eficiencias adiabáticas del compresor y la turbina son del $100 \%$.

En el Cuadro 2 se muestran como cambian los valores de la relación de presiones, trabajo neto y relación de trabajo de retroceso para aire como sustancia de trabajo y diferentes temperaturas de entrada a la turbina $T_{3}$ y $T_{1}=$ $300 \mathrm{~K}$. Un incremento de la temperatura máxima $T_{3}$ ocasiona una disminución en la relación de trabajo de retroceso y un aumento en el valor del trabajo máximo para una misma sustancia de trabajo. Cuando la temperatura de entrada a la turbina $T_{3}=1500 \mathrm{~K}$, el trabajo óptimo es de $W_{\text {neto }} 237.1 \mathrm{kj} / \mathrm{kg}$ y la relación de acoplamiento $W_{C} / W_{T}=57.85$ pero para $T_{3}=2500 \mathrm{~K} \mathrm{el} \mathrm{máximo}$ corresponde a $W_{\text {neto }}=704.6 \mathrm{kj} / \mathrm{kg}$ y $W_{C} / W_{T}$ $=45.08$. En las figuras 8 y 9 se muestra como varía la relación de trabajo de retroceso cuando se modifican las temperaturas máximas y se observa un incremento en el trabajo neto y un desplazamiento del punto óptimo hacia regiones donde es menor la relación de acoplamiento. En todos los casos se ha considerado que las eficiencias adiabáticas del compresor y la turbina son del $75 \%$ y $80 \%$, respectivamente.

De acuerdo con (27) para un ciclo Brayton con irreversibilidades la relación de trabajo de retroceso máxima $r_{\text {trmáx }}$ sólo dependerá de las temperaturas máximas y mínimas del ciclo, de las eficiencias adiabáticas de la turbina y el compresor y es independiente de la sustancia de trabajo. El valor máximo que alcanza la relación de acoplamiento turbina-compresor siempre es la misma aún cuando sea diferente la sustancia de trabajo utilizada, lo que concuerda con la expresión (27).

\section{CONCLUSIONES}

El estudio de la ecuación deducida es capaz de predecir las condiciones de operación que permiten obtener una relación de acoplamiento máxima en un ciclo Brayton.

Aun cuando los valores de $r_{t r}$ son función del coeficiente $\gamma$ de la sustancia de trabajo, de las temperaturas de entrada al compresor y a la turbina, de las eficiencias del compresor y la turbina y de la relación de presiones $r_{p}$, el valor máximo de $r_{t r}$ se mantiene constante siempre que $T_{1}, T_{3}, \eta_{T}, \eta_{C}$ y se mantengan fijos.

Para un ciclo Brayton con irreversibilidades, altos valores de la relación $T_{1} / T_{3}$ incrementan la relación de trabajo de retroceso máxima, siempre que se mantengan fijos las eficiencias adiabáticas del compresor y la turbina.

Para procesos de compresión y expansión isentrópicas donde las eficiencias adiabáticas son del $100 \%$,la relación de trabajo de retroceso máxima presenta una disminución siempre que la relación $T_{1} / T_{3}$ se mantenga constante. 


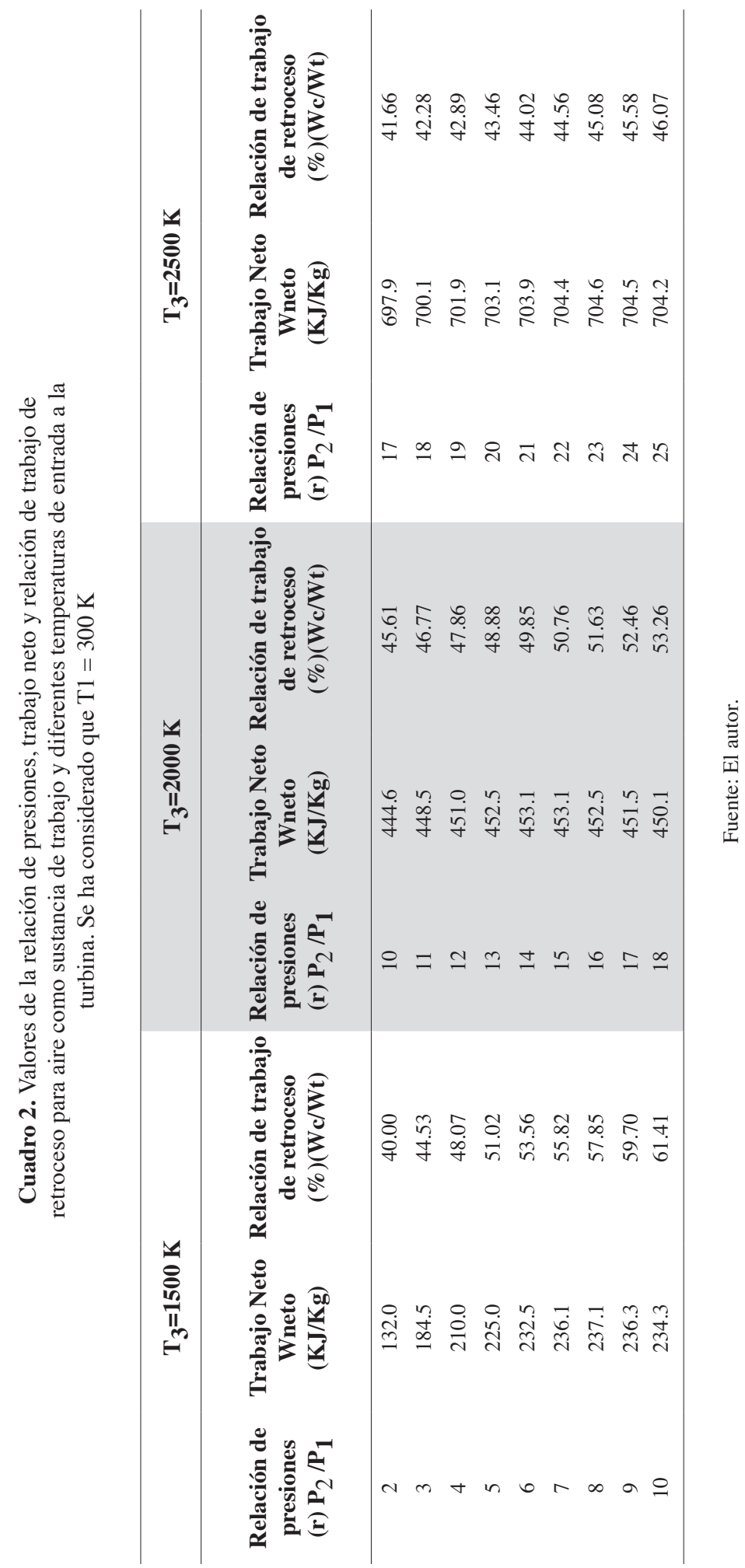




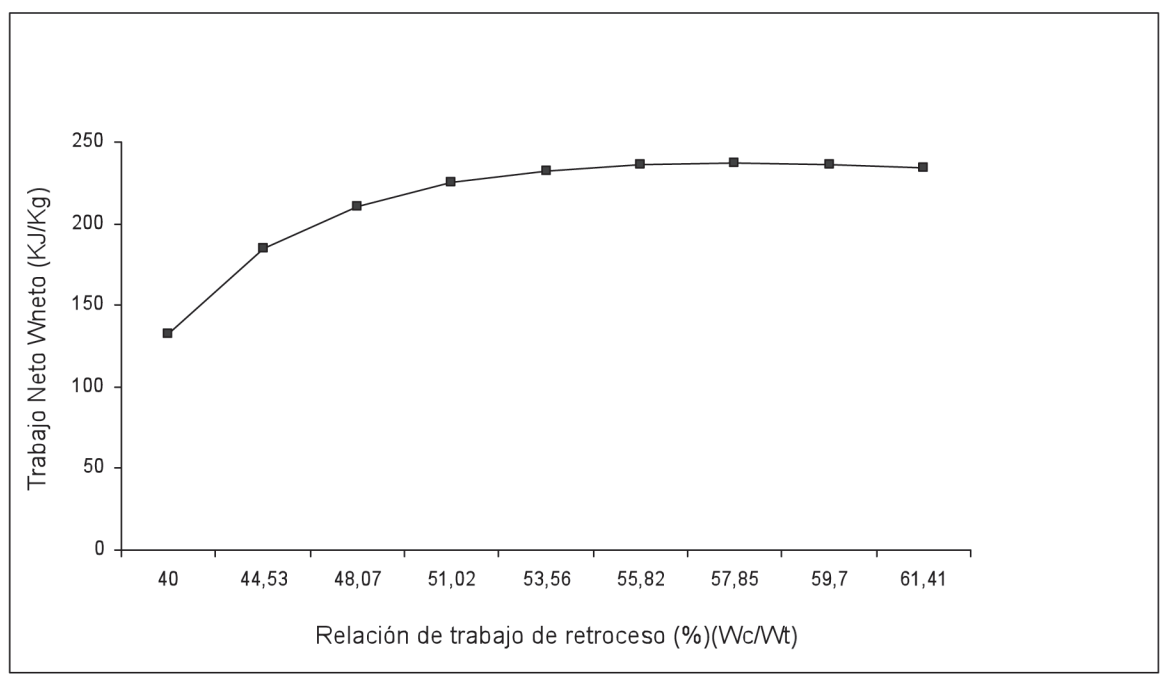

Figura 8. Variación del trabajo neto en $\mathrm{KJ} / \mathrm{Kg}$ versus la relación de trabajo de retroceso para el aire. Se ha considerado que $\mathrm{T}_{3}=1500 \mathrm{~K} \mathrm{Y} \mathrm{T}_{1}=300 \mathrm{~K}$.

Fuente: El autor.

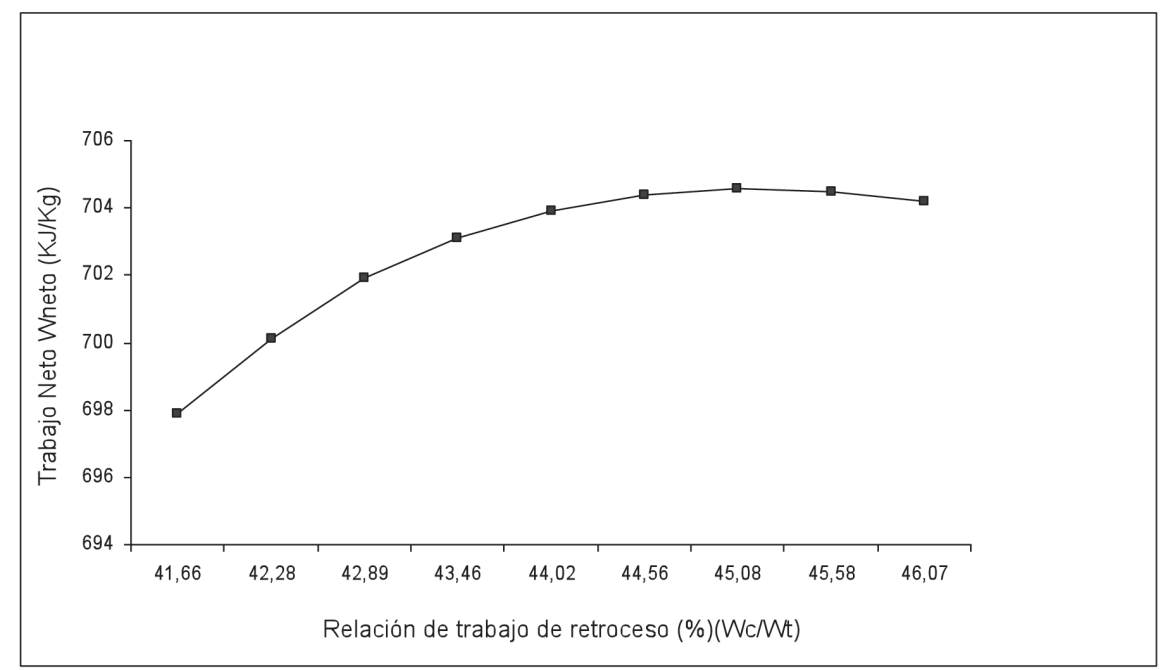

Figura 9. Variación del trabajo neto en $\mathrm{KJ} / \mathrm{Kg}$ versus la relación de trabajo de retroceso para el aire. Se ha considerado que $\mathrm{T}_{3}=2500 \mathrm{~K} \mathrm{Y} \mathrm{T}_{1}=300 \mathrm{~K}$.

Fuente: El autor. 


\section{REFERENCIAS BIBLIOGRÁFICAS}

Burghardt, M.D. (1984). Ingeniería Termodinámica. México: Editorial Harla.

Cengel, Y. y Boles, M. (2006). Termodinámica. México: McGraw-Hill Interamericana.

Chen, L., Zheng, J., Sun, F. y Wu, C. (2002). Performance comparison of an irreversible closed Brayton cycle under maximum power density and maximum power conditions. Exergy, an International Journal, 4(2), 345-351.

Curzon, F.L y Ahlborn, B. (1975). Efficiency of a Carnot Engine at maximum power output. Am.J.Phys, 43(22), 22-24.

Guzmán-Vargas, L. y Angulo-Brown, F.(1998). A dissipative Joule-Brayton cycle model. Rev. Mex.Fís, 44(6), 619-623.

Herrera, C.A, Rosillo, M.E y Castaño, L. (2008). Fugas de calor y aprovechamiento de efluentes en la optimización de ciclos Brayton totalmente irreversibles. Rev.Mex. Fís , 54(2), 118-126.

Jones, J.B. y Dugan,R.E.(1997). Ingeniería Termodinámica. México, D.F: Prentice-Hall Hispanoamérica, S.A.

Leff, H. (1987). Thermal efficiency work output: New results for old heat engines. Am.J.Phys, 55(7), 602-610.
Malaver, M. (2008). Análisis de las temperaturas, la eficiencia térmica y el trabajo neto en un ciclo dual. Educ. Quím, 19(2), 122-126.

Russel, L.D. y Adebiyi, G.A.(1997). Termodinámica Clásica. Delaware: Addison -Wesley Iberoamericana, S.A.

Urrecheaga, K. y Malaver, M.(2003). Análisis de las temperaturas y de la eficiencia térmica en el ciclo Otto. Educ. Quím, 14(3), 138-141.

Van Wylen, G.J., Sonntag, R.E. y Borgnakke, C.(2000). Fundamentos de Termodinámica. México: Editorial Limusa, S.A.

Wark, K.J. y Richards, D.(2001). Termodinámica. Madrid: McGraw-Hill Interamericana, S.A.

Woodyard, D. (2004) Pounder's Marine Diesel Engines and Gas Turbines. Elsevier ButterworthHeinemann.

\section{SOBRE EL AUTOR}

\section{Manuel Malaver}

Licenciado en Química. Doctor en Mecánica Teórica y Aplicada. Docente e investigador en la Universidad Marítima del Caribe en la escuela de Naútica e Ingeniería en el departamento de Ciencias Aplicadas.

Teléfono: 0058-212-3501066

Correo electrónico: mmf.umc@gmail.com 
